\title{
The signatures of magnetic reconnection according to the microwave drifting bursts observations
}

\author{
N. S. Meshalkina ${ }^{1} \dagger$, A. T. Altyntsev ${ }^{1}$, \\ V. V. Grechnev ${ }^{1}$, \\ and Yan Yihua ${ }^{2}$ \\ ${ }^{1}$ Institute of Solar-Terrestrial Physics, Lermontov st. 126, Irkutsk, Russia 664033 \\ email: nata@iszf.irk.ru \\ ${ }^{2}$ National Astronomical Observatories, Chinese Academy of Sciences, Datun Road A20, \\ Chaoyang District, Beijing 100012, China \\ email: yyh@bao.ac.cn
}

\begin{abstract}
A series of drifting microwave bursts during the 30 March 2001 flare are analyzed using the Siberian Solar radiotelescope (SSRT) images at $5.7 \mathrm{GHz}$ and dynamic spectra obtained simultaneously by the spectropolarimeters of National Astronomic Observatories in China (NAOC) in the range 5.2-7.6 GHz. While observing the event with the SSRT, the burst sources were simultaneously recorded at two frequencies, which allowed their relative spatial shifts to be measured and source velocity along the flare loop (observed in soft X-ray and ultraviolet emission) to be evaluated. Estimates were made of the plasma density gradient along the source movement direction, the plasma emission being assumed to be generated at the second harmonic. Drifting burst series occur during transient hard X-ray brightenings. Burst drift rates ranged from -10 to $20 \mathrm{GHz} / \mathrm{s}$, with a mean value of about $6 \mathrm{GHz} / \mathrm{s}$. The shape of the drift rate distribution around the mean value is nearly symmetric. It is suggested that the mean value distribution may be related to increased plasma density in the source of subsecond pulses. In particular, the corresponding density variations may be associated with magnetic reconnection processes.
\end{abstract}

Keywords. Solar flares, microwave radiation, magnetic reconnection

\section{Discussion}

When the drifting bursts are generated by a plasma mechanism the frequency drift velocity can be determined as:

$$
\frac{\partial f}{\partial t}=\frac{A}{\sqrt{n}} \frac{\partial n}{\partial t}=\frac{A}{\sqrt{n}}\left(\frac{\partial n}{\partial t}+\frac{\partial n}{\partial l} \frac{\partial x}{\partial t}\right)=\frac{A}{\sqrt{n}}\left(\frac{\partial n}{\partial t}+\frac{\partial n}{\partial l} v\right) \approx 2 \triangle f\left(\frac{1}{\tau_{\mathrm{ssp}}}-\frac{v}{\triangle l}\right)
$$

where $A=\frac{\alpha}{2 \pi} \sqrt{\frac{4 \pi n e^{2}}{m}}$ with $\alpha=1$ for the Langmuir frequency, and $\alpha=2$ for the harmonic, $\triangle f=(5.78-5.69) \mathrm{GHz}=0.09 \mathrm{GHz}, \tau_{\mathrm{ssp}}$ is temporal scale of plasma density variation due to MHD processes, $v$ is velocity of emitting electrons, $\Delta l$ is observed displacement of source centroids at $5.78 \mathrm{GHz}$ and $5.69 \mathrm{GHz}$. Usually, to interpret frequency drift rates, only the second term in parenthesis is considered. This term accounts for the plasma density gradient $\partial n / \partial l$ along the propagation path. The first term, representing density changing in the emission region due to dynamic MHD processes, has not been considered before.

$\dagger$ Present address: Institute of Solar-Terrestrial Physics, Lermontov st. 126, Irkutsk, Russia. 
This circumstance along with an obvious symmetry in the scattering of frequency drift rates around the mean value of $6 \mathrm{GHz} / \mathrm{s}$ prompt us to take into account the term $2 \triangle f \frac{1}{\tau_{\mathrm{ssp}}}$ and to estimate it as $6 \mathrm{GHz} / \mathrm{s}$. After this correction of the observed drift velocities the calculated velocities of exciters do not exceed the velocity of light, and vary over a reasonable range. So, there are serious reasons to suggest the essential contribution of dynamic density variations to the observed frequency drift values.

To study mechanisms of impulsive generation we have to consider the non-stationary model, since transit time $L / V_{A}>2 \mathrm{sec}$, if the sheet width is order of the observed displacements : $\mathrm{L}=2 \operatorname{arcsec}(1450 \mathrm{~km})$ and $V_{A}=6.4 \times 10^{7} \mathrm{~cm} / \mathrm{s}$. We should note that close estimate of acceleration region size $(2000 \mathrm{~km})$ was obtained from the study of the decimetric pulses (Aschwanden, et al. (1993)).

For large $L / V_{A}$ the increasing of the current sheet density can be estimated in one dimensional approximation as

$$
\frac{\partial n}{\partial t}=\frac{n u}{\triangle}\left(1-\frac{\left(t-t_{0}\right)}{\triangle} \frac{\partial \triangle}{\partial t}\right)
$$

where $\triangle$ is current sheet thickness. The first term in parenthesis describes plasma inflow with velocity from outside the current sheet, and the second is related to changing current sheet thickness. The strength of electric field, excited by reconnection in a sheet should exceed the Dricer field value for particle acceleration to occur. For this case the estimate of reconnection velocity or inflow plasma velocity $u \geqslant 0.1 V_{A}, \mathrm{~cm} / \mathrm{s}$. This value conforms to standard estimations in flares processes (Priest and Forbes (2000)). With this value of $u$ the density growth corresponding to the drift rate of $6 \mathrm{GHz} / \mathrm{s}$ can be achieved for current sheet with thickness of $50 \mathrm{~km}$. It was proposed a model with dynamic reconnection - the formation of magnetic islands inside a current sheet with their subsequent merging - for interpreting the impulsive decimetric structures (Kliem, et al. (2000)). For sheet parameters obtained above, period of island forming due to tearing instability considerably exceeds the burst duration.

The displacements of drifting sources in the cm-range were measured using the observation data simultaneously recorded at two frequencies with spatial resolution, which permits one to estimate independently both the plasma density gradient along the source movement and emission source velocity. The hypothesis was suggested connecting this character of scattering with the effect of dynamic density increase in subsecond pulse generation region. In particular the estimations were made explaining observed density increase by magnetic reconnection process if the velocity of magnetic reconnection is about $0.1 V_{A}$.

\section{Acknowledgements}

This work was supported by the Russian projects of RFBR 04-02-39003, 05-07-90147.

\section{References}

Aschwanden, M., Benz, A.O., and Schwartz, R.A., 1993, ApJ 417, 790.

Kliem, B., Karlicky, M., and Benz, A.O., 2000, Astronomy \& Astrophysics 360, 715.

Priest, 2000, Priest, E. and Forbes, T., Magnetic Reconnection, Cambridge, UK: Cambridge University Press, June 2000., p. 612. 